\title{
¿Por Defecto o POR Defectos? \\ LAS CAUSAS DE LA DESCONFIANZA \\ institucional en AmÉrica Latina
}

\author{
José Del Tronco ${ }^{1}$
}

Resumen: ¿Bajo qué condiciones los ciudadanos desconfían de las instituciones políticas? Las respuestas a esta pregunta son variadas y pueden ubicarse en dos grandes corrientes: a) la culturalista que destaca la importancia de los valores culturales, y b) la racional culturalista, según la cual la desconfianza es resultado de los deficientes desempeños de los gobiernos democráticos. La presente investigación analiza tres diferentes dimensiones de la performance, vinculadas todas ellas al concepto de representación y relacionadas con etapas, actividades y actores específicos: 1 . la calidad institucional (relativa a las reglas de juego y su funcionamiento); 2. la calidad de la política (relativa a los a la calidad de los procesos de deliberación, negociación y sanción de leyes, así como a la productividad legislativa); 3. la calidad de los resultados (vinculada al impacto de las políticas públicas sobre el bienestar social). Con datos de Latinobarómetro 2006, el análisis demuestra que los latinoamericanos tienden a confiar más cuanto mejor es el desempeño gubernamental, pero ello no obedece sólo a indicadores económicos sino también a la evolución de las condiciones políticas e institucionales

Doctor en Ciencia Política con Mención de Honor por la Universidad Nacional Autónoma de México. Profesor Investigador de tiempo completo en la Facultad Latinoamericana de Ciencias Sociales Sede Académica de México. Carretera al Ajusco 377, Col. Héroes de Padierna. Delegación Tlalpan, México D.F. +52 553000 0200. Email: jdeltronco@flacso.edu.mx 
Palabras clave: Confianza institucional, representación, cultura política, América Latina

Abstract: Under which conditions citizens distrust on political institutions? Answers to these questions are diverse and may be classified in two main approaches: culturalist and rational culturalist. Based on the latter, this paper analyzes the association between quality of political representation and institutional confidence in Latin America. This research is focused on the concept of representation and its dimensions (rules, élites and government effectiveness), and support the hypothesis that people confidence on political institutions is better explained by the performance of representative actors than cultural values of people. With data obtained from 2006 Latinobarometer survey, empirical findings show that Latin American citizens willingness to trust depends on government performance, but they are worried not just by economic indicators. Institutional and political conditions are also quite important.

Key words: Institutional trust, representation, political culture, Latin America

\section{La desconfianza política en América Latina}

Una enorme cantidad de estudios han remarcado durante los últimos años los crecientes niveles de escepticismo y desconfianza que los ciudadanos de diversas regiones del mundo expresan respecto de sus autoridades políticas (Torcal y Montero, 2006; Inglehart y Wezel, 2005; Almond et al., 2002; Putnam et al., 1999; Norris, 1999). En el caso de las democracias consolidadas, este fenómeno abarca el último cuarto del siglo XX. En los Estados Unidos de América, país cuyo acervo estadístico permite analizar la evolución de la opinión pública en una "larga duración", dicho declive ha sido más profundo a partir de la Guerra de Vietnam y se ha mantenido durante las últimas cuatro décadas (Nye, 1998; Putnam, 2000). En el caso de Europa 
Occidental, este fenómeno es más reciente y se expande durante los años ochenta y noventa (Newton, 1999; Miller y Listhaug, 1999; Klingemann, 1999).

Por su parte, en los países de reciente democratización, los estudios al respecto no han podido confirmar una tendencia clara (Evans y Letki, 2006; Gunther y Montero, 2006; Catterberg y Moreno, 2005; Torcal 2006). Muy probablemente a raíz del cambio profundo que implicó la liberalización política en buena parte de Europa del Este y América Latina, la evidencia sobre el estado general del sentimiento público -especialmente para el caso latinoamericano- está aún en construcción y sus factores explicativos no están del todo claros. Mientras que los análisis de la situación en Latinoamérica tienden a destacar el malestar de los ciudadanos para con sus representantes (Gargarella, 2002), los estudios sobre el tema en los países ex-comunistas señalan una tendencia general de los ciudadanos a expresar sentimientos de desconfianza y cinismo respecto de las nuevas instituciones políticas y sociales. Mishler y Rose (1997), por caso, señalan que entre los ciudadanos de países pos-comunistas, sólo un $31 \%$ expresa confianza en las instituciones, $22 \%$ son escépticos, y el $47 \%$ restante es claramente desconfiado (distrustful) respecto de las mismas.

En América Latina, los procesos de democratización experimentados durante las últimas tres décadas han puesto al tema de la "confianza ciudadana en las instituciones políticas" en el centro del debate. A diferencia de la legitimidad democrática ${ }^{2}$-que es significativa entre los

La concepción tradicional de legitimidad deriva de Weber, y refiere a aquellas actitudes de aquiescencia de parte de los ciudadanos a una determinado tipo de dominación política. En este caso, y derivado de allí, la legitimidad implica asimismo, una valoración positiva; es decir, la dimensión normativa (de lo que "debe ser" un régimen político) está presente en su definición. Es lo que Easton denominó “apoyo político 
latinoamericanos- y de la satisfacción con el desempeño del régimen ${ }^{3}$-que es oscilante y parece depender en buena medida de la cercanía ideológica con el gobierno de turno (Torcal, 2001)-, las y los latinoamericanos expresan (con diferencias entre países) sentimientos generalizados de alienación y cinismo ${ }^{4}$ respecto de la política y lo político. Si bien la democracia sigue siendo el sistema político preferido en la región (según el Latinobarómetro, entre 1996 y 2009, dos de cada tres latinoamericanos consideraba a la democracia como el mejor sistema de gobierno posible más allá de sus problemas), los niveles de confianza en las instituciones centrales de la democracia representativa como los partidos políticos o el parlamento-se mantienen comparativamente bajos y de manera bastante paradójica tienden a caer en todos los países del continente a medida que las democracias adquieren más edad.

difuso" (1974) porque no está condicionado al desempeño de las instituciones políticas.

3 En el caso de la satisfacción, ella refleja una actitud de tipo cognitivo, que es expresión ya no de una dimensión normativa sino del nivel de bienestar asociado al funcionamiento de un determinado tipo de régimen o gobierno. La satisfacción, por tanto, en términos de Easton, representaría un tipo de "apoyo político específico", en la medida que es de corto plazo y depende del funcionamiento efectivo de las instituciones de gobierno. (Enciclopedia Blackwell, disponible en línea en el siguiente vínculo: http://www.blackwellreference.com/public/ tocnode?id=g9781405131995_chunk_g978140513199521_ss55-1)

El cinismo -como actitud- tiene lugar cuando una persona no espera nada de los demás, porque supone que la conducta humana sólo está motivada por su propio interés. Cappella y Jamieson (1997: 166) definen el cinismo político como "la desconfianza generalizada de los líderes o grupos políticos con el proceso político como un todo" por corromper a los personas que participan en él y que atrae a las personas corruptas como participantes. El cinismo como la falta de confianza ha sido tratado a nivel de las instituciones de gobierno y el régimen en su conjunto (por ejemplo en Miller, 1974). 
Figura 1: Actitudes hacia la democracia en América Latina (1996-2009) ${ }^{5}$

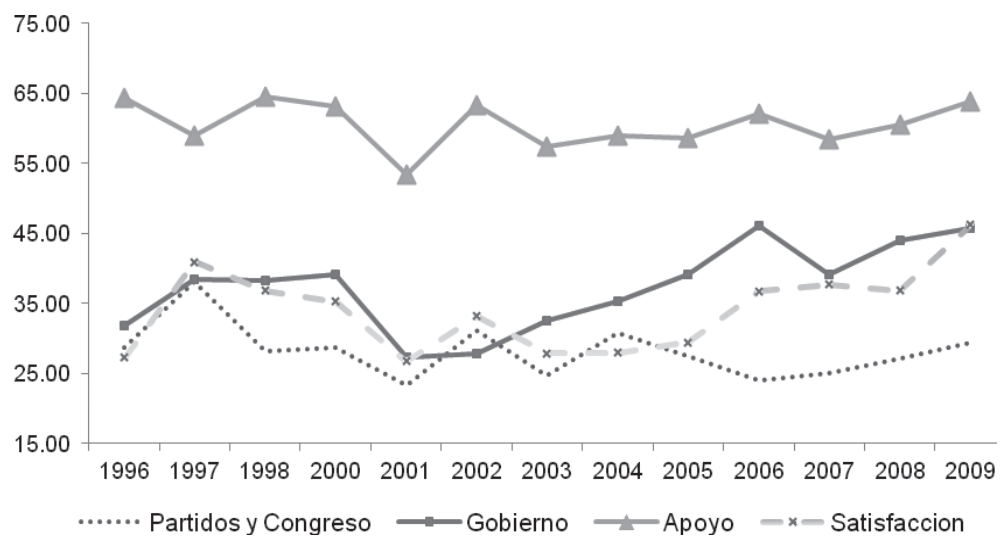

Fuente: Elaboración propia en base a Latinobarómetro (199697-98-2000-01-02-03-04-05-06-07-08-09)

En América Latina, los bajos niveles de confianza institucional son consistentes con diversos marcos teóricos. Para los teóricos de la modernización, la desconfianza es producto del cambio de valores sociales predominantes, producido por las nuevas pautas de consumo y relacionamiento social en las sociedades complejas (Dalton, 1999; Dalton et al., 2002). La individualización y el desprestigio de las autoridades tradicionales, motiva -especialmente en las nuevas generaciones- un sentimiento de desconfianza hacia las instituciones políticas (Inglehart y Wezel,

\footnotetext{
El "apoyo a la democracia" o "political support" refiere a la valoración normativa o legitimidad del régimen democrático frente a otros. La satisfacción con el funcionamiento de la democracia es parte de la dimensión cognitiva del apoyo expresado por ciudadano a un determinado régimen político, mientras que la confianza -en este caso, hacia partidos, congreso y gobierno- es una de las variables constitutivas de la dimensión "afectiva".
} 
2005). Por su parte, la corriente culturalista más tradicional sostiene que no es el cambio cultural sino más bien la persistencia de valores no democráticos (evidenciados por siglos de historia política autoritaria y bajos niveles de capital social entre su población) el elemento determinante de su desconfianza hacia las nuevas instituciones (Torcal, 2006). Finalmente, la hipótesis racional-culturalista sostiene que la desconfianza hacia las instituciones y actores políticos se explica por el deficiente desempeño de éstos últimos con relación a las expectativas generadas durante la transición (Mishler y Rose, 2001).

No obstante la proliferación de posibles explicaciones, no hay acuerdo sobre las verdaderas causas de la desconfianza institucional. Para el caso latinoamericano, la ausencia de análisis transversales obliga a cuestionarse: ¿bajo qué circunstancias los ciudadanos desconfían de los actores e instituciones políticas en contextos democráticos? ¿Cuáles son sus particularidades en el caso de la región latinoamericana, y para cada uno de los países que la componen? ¿Hay diferencias significativas entre las instituciones (partidos, parlamento, gobierno y presidente) o es posible establecer perfiles actitudinales de los ciudadanos igualmente válidos para todas ellas?

El desarrollo del documento, a continuación, se orienta a dar respuesta a dichos interrogantes. En el segundo apartado, se presentan las distintas corrientes que han intentado dar una respuesta a esta pregunta, y se postula y desarrolla -brevemente- la adoptada por este trabajo. En la tercera sección, se presenta el análisis empírico. En primer término, se hace referencia al proceso de operacionalización; se establecen las dimensiones de análisis y sus indicadores. A continuación, se presenta los resultados obtenidos a partir de los análisis estadísticos -en sus distintos niveles de análisis: agregado por países e individual- entre 1996 y 
2006. ${ }^{6}$ En la sección final, se exponen algunas conclusiones y sus implicaciones para el estudio de las democracias en la región.

\section{La desconfianza política: relevancia e hipótesis causales}

La relevancia de la confianza institucional se explica porque es el elemento que hace posible la delegación de responsabilidades de los ciudadanos hacia sus representantes. En tal sentido, sin una dosis de confianza inicial, la representación no puede tener lugar (Pitkin, 1985). Adicionalmente, y en términos de su contribución a la estabilidad del régimen político, la confianza institucional evita el desligamiento de los ciudadanos respecto de sus representantes y nutre a estos últimos de proyectos políticos capaces de definir y abordar los problemas públicos de manera consistente con las preferencias de aquellos. Dota a la democracia representativa, en definitiva, de sus propios fundamentos (Mouffe, 2007).

Conceptualmente, este trabajo define a la confianza como la esperanza o nivel de certidumbre de una persona o grupo de personas sobre el comportamiento de una contraparte. Cuando ésta actúa de acuerdo a las expectativas de la primera, la confianza aumentará y viceversa (Locke, 1999). De acuerdo con esta definición, la confianza es siempre un término probabilístico por ser función o resultado de distintas variables.

El motivo por el cual se eligió el año 2006 es porque constituye la medición más cercana y menos influida por la crisis económica mundial iniciada en 2008 -cuyos primeros indicios se manifestaron ya a finales de 2007- y aislar así la incidencia de factores extraordinarios sobre el objeto de estudio 
La desconfianza es la otra cara de la misma moneda. En situaciones en que la certidumbre acerca del comportamiento ajeno es alta -ya por la experiencia pasada, ya por la conocida efectividad de la sanción a conductas oportunistas-, el incumplimiento de la contraparte puede no generar desconfianza de forma inmediata. Esta última surge como resultado de incumplimientos sistemáticos que erosionan la certidumbre original, resultando en niveles de esperanza cada vez menores. En estos casos, sociedades con muy bajos niveles de confianza institucional, una conducta no esperada de las contrapartes genera desconfianza con mayor rapidez; basta un número reducido de incumplimientos (sólo uno, en ocasiones) para que aquella tenga lugar. ${ }^{7}$

Desde la intuición, parece posible explicar por qué dicha confianza está ausente cuando la dominación ejercida por los gobiernos adquiere un formato autoritario $o$ dictatorial. En esos casos, no existe ningún mecanismo institucional que garantice la representación de los intereses de los ciudadanos, y de los grupos sociales que éstos conforman. Sin embargo, durante las últimas décadas muchas democracias (regímenes que sí cuentan con dicha garantía), y entre ellas una buena cantidad de países latinoamericanos, han visto descender de manera continua y pronunciada los niveles de confianza de sus ciudadanos en las instituciones políticas que los representan. La paradoja es clara: ¿Por qué los ciudadanos desconfían de las instituciones políticas representativas cuando -en teoría- ellos

Tal como lo señaló Newton (1999), confianza social y confianza política son dimensiones diferentes. La primera puede ser anónima o personalizada y tiene por objeto a las personas; nuestros semejantes, en sus distintos roles sociales. La confianza política, por su parte, es aquel activo que los individuos depositan en las instituciones y actores de gobierno a través del proceso de delegación. Ésta última es el objeto del presente estudio. 
mismos las han diseñado y a sus integrantes han elegido para representar sus intereses?

La literatura especializada ha presentado diferentes respuestas a este problema, que se agrupan aquí en dos grandes corrientes. La perspectiva culturalista sostiene que las actitudes políticas son resultado del proceso de socialización (Almond y Verba, 1959; Eckstein, 1988; Inglehart, 1988; Putnam, 1993; Torcal, 2006; Segatti, 2006) y por lo tanto, exógenas al sistema político. Desde esta perspectiva, la desconfianza institucional podría explicarse a partir de la persistencia de patrones culturales de largo plazo que han permeado el funcionamiento de los regímenes políticos modernos hasta la fecha. Siglos de instituciones autoritarias -y algunas décadas de experiencias democráticas fallidas e inestables- han marcado culturalmente a los ciudadanos, y permeado sus actitudes políticas hasta la actualidad. De acuerdo con esta visión, los patrones culturales así como las experiencias democratizadoras originales (Torcal, 2006) conducirían a los ciudadanos latinoamericanos o bien a preferir las pautas autoritarias o bien -en caso de considerar negativamente esta última opción- a adoptar un concepto particular de democracia; menos centrado en los procedimientos liberales de representación política, y más asociado a la vigencia de decisores poderosos que establecen una relación directa -sin las clásicas intermediaciones institucionales- con los ciudadanos ("el pueblo"). En este contexto, toda democracia liberal, establecida a partir del rol central de las instituciones de intermediación (partidos políticos, fundamentalmente) chocaría con la desconfianza de los ciudadanos para quienes dicho arreglo institucional constituiría una clara deformación de la pauta democrática mayoritaria o republicana. 
Por su parte, la tesis racional-culturalista plantea que si bien los patrones culturales juegan su papel, es el desempeño de los gobiernos el elemento que explica de manera significativa los cambios en las actitudes políticas de los ciudadanos, y especialmente, en los niveles de confianza en las instituciones representativas (Mishler y Rose, 2001). De acuerdo con este enfoque, la cultura política es un ingrediente importante, y las actitudes de los ciudadanos contienen información relevante acerca del funcionamiento pasado y futuro de los sistemas políticos. Sin embargo, para los racionalistas, la huella cultural es menos profunda y las actitudes políticas cambian en el corto plazo como resultado del desempeño "percibido" de las instituciones representativas (Camoes, 2000; Miller y Listhaug, 1999).

Este trabajo adopta el enfoque "racional". De acuerdo con esta corriente, los ciudadanos son individuos racionales capaces de juzgar consistentemente la utilidad de una institución, sus consecuencias distributivas y sus efectos sobre el bienestar individual (North, 1993; Knight, 1996), y sus actitudes políticas, formadas en buena medida durante el proceso de socialización, lejos de ser estables pueden cambiar a partir del funcionamiento del sistema político (Lane, 1992). Para el caso latinoamericano, dado que la transición a la democracia marca el inicio de un juego de representación deseado por la mayor parte de los ciudadanos, esta investigación propone que la desconfianza "política" (Newton, 1999) es, en buena medida, un reflejo del desempeño de los actores políticos y de las instituciones representativas en relación a lo que se espera de ellas; esto es, cuanto mayor la distancia entre el desempeño percibido y el esperado, mayor la desconfianza de los ciudadanos hacia las instituciones propias de la democracia representativa. 
El mecanismo causal que vincula a la representación con la desconfianza es la percepción que los ciudadanos tienen del desempeño de sus representantes; es decir el grado a través del cual los ciudadanos perciben que sus preferencias están presentes en las decisiones políticas. En pocas palabras, los niveles de confianza reflejan que tan nítidamente se hacen presentes -en la arena política formal- las preferencias de los mandantes durante el proceso de selección, diseño, implementación y evaluación de políticas. En tal sentido, es lógico esperar que los ciudadanos (con la información siempre incompleta de que disponen) depositen su confianza en determinados actores políticos en función de un juicio retrospectivo (Fiorina, 1981).

\section{La calidad de la representación y sus dimensiones como factor explicativo}

En términos políticos, la representación es un proceso a través del cual las voces, opiniones, perspectivas e intereses de los actores políticos (ya sean individuales o colectivos) "se hacen nuevamente presentes" (se re-presentan) en el proceso de toma de decisiones. Así, la representación ocurre cuando los "representantes" hablan, abogan y actúan en nombre de otros -sus representados- en la arena política (Pitkin, 1985).

De acuerdo con los trabajos clásicos de representación (Pitkin, 1985; Manin, Stokes y Przeworski, 1998), existen diferentes maneras de abordar el fenómeno representativo, que podrían resumirse en las siguientes: i) en primer lugar, la representación como autorización o como "voto de confianza", refiere a un proceso en el cual los ciudadanos delegan en su(s) hombre(s) de confianza la responsabilidad de hablar en su nombre, sin más condicionamientos que su 
propio criterio; ii) la representación como responsabilidad, por su parte, supone que el representante es un "agente" que se compromete a dar cumplimiento a los mandatos de su "principal". En el primer caso, los representantes

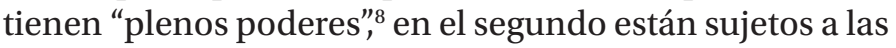
instrucciones de sus mandantes. En el primer caso, una representación de calidad está dada por los resultados del proceso de representación; es decir por el impacto de las políticas. En el segundo, no importan sólo los resultados sino también el cumplimiento de los mandatos, esto es, el contenido de las políticas. ${ }^{9}$

A diferencia de ambas visiones, este trabajo propone entender y analizar la calidad de la representación desde un enfoque amplio, que incluye dimensiones institucionales, políticas y de políticas públicas (Figura 2).

8 De allí el nombre que recibían, por ejemplo, los ministros "plenipotenciarios" de las nacientes repúblicas americanas cuando, a principios del siglo XIX, negociaban las condiciones de los acuerdos internacionales entre ellos o ante los representantes de potencias europeas). Como no podían estar consultando a sus gobiernos dada la ausencia de facilidades para una comunicación inmediata, partían con instrucciones -más o menos precisas- respecto de los resultados, pero tenían "plenos poderes" para negociar las condiciones en que tales acuerdos resultarían factibles.

$9 \quad$ El problema en este caso radica en el dilema que se genera cuando una vez en el cargo, los representantes llegan a la conclusión de que cumplir con lo (com)prometido puede acarrear más pérdidas que beneficios. Por ello algunos autores sostienen que la ausencia de mandato imperativo obliga a los representantes a cambiar de rumbo y buscar el mayor beneficio social, aunque ello contravenga los acuerdos pre-electorales (Stokes, 1999 y 2002). 
Figura 2: Dimensiones de la representación durante el ciclo de políticas

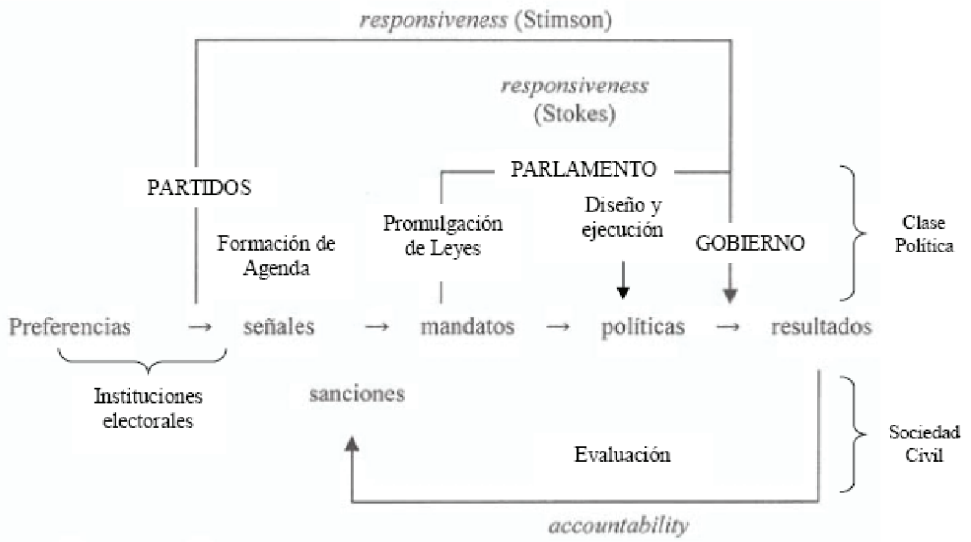

Fuente: Elaboración propia en base a Manin (1999: 9)

$\mathrm{Si}$, tal como fue previamente establecido, la representación es el proceso a través del cual determinados delegados hablan en nombre de los ciudadanos y de esa forma consiguen satisfacer sus intereses, la calidad del proceso de representación política es el resultado de tres fenómenos o dimensiones: 1) si quienes hablan en nombre de la comunidad política son efectivamente aquellos que la comunidad política ha elegido; 2) si lo que dicen es lo que efectivamente diría cualquiera de sus representados en su lugar, y 3) si los resultados de dicho proceso satisfacen los intereses de los representados. Dicho en otros términos, las dimensiones de análisis refieren a la calidad de los procedimientos institucionales (polity), que hacen posible la selección de representantes, la calidad de la política (politics), que refiere a la capacidad del sistema político por incorporar al proceso de formulación de políticas las 
preferencias de los votantes, y, finalmente, la calidad de las políticas públicas (policies), a través de cuyos resultados se puede observar en qué medida están representadas las preferencias de los ciudadanos.

\section{a) La dimensión institucional: Estado de Derecho y reglas electorales}

Una primera dimensión de la calidad de la representación permite analizar la capacidad de las instituciones para representar de manera efectiva la distribución de preferencias al interior de una sociedad. Desde este enfoque, se parte del supuesto de que las instituciones importan, pero hay diferentes tipos y niveles de instituciones. En esta primera dimensión, nos interesan aquellas garantizan determinados derechos políticos y libertades civiles, así como la selección libre, competida, equitativa y proporcional, de los representantes por parte de los ciudadanos (Leiras s/f; Levine y Molina, 2006; Morlino, 2005; O’Donnell et al., 2004; Bingham Powell, 2004; Przeworski, 1995) ${ }^{10}$.

\section{b) La dimensión política: Sistemas de partido y calidad de la deliberación parlamentaria}

Un segundo abordaje para analizar el grado de representatividad de las instituciones democráticas -desarrollado a lo largo de la última década por Manuel Alcántara et al. (2006) - considera relevante analizar la calidad de la política, a partir de las características y el desempeño de las élites. Esto implica tomar en cuenta no sólo las características del entramado institucional que encarna y da forma al proceso de representación sino también a los actores políticos que la hacen posible. En este caso, se parte del

10 Para una revisión importante de estas dimensiones en América Latina, ver Payne, Zovatto y Mateo Díaz (2006). 
supuesto de que estos últimos (en tanto representantes) constituyen un actor clave que define el contenido y las formas en que las políticas públicas son incorporadas a la agenda y formuladas, posteriormente, para su implementación. ${ }^{11}$ El funcionamiento efectivo de las asambleas y la actividad de los representantes se vinculan al cumplimiento de los mandatos electorales; su sesgo ideológico y el honor a las promesas de campaña en detrimento de la maximización de sus rentas individuales en el marco de dichas instituciones. No se trata de ignorar al conjunto de normas formales que regulan la interacción humana, sino de analizar -también- a los actores que cotidianamente se mueven en la arena político-institucional (Alcántara Sáez, 2006). ${ }^{12}$

\section{c) Los resultados de las políticas públicas como indicador de calidad}

Las dos primeras dimensiones del proceso de representación hacen hincapié en el tema de los procedimientos

11 Es la política como actividad de deliberación, argumentación y negociación la que está en el centro de dichas dimensiones. Un análisis interesante para el caso mexicano puede encontrarse en Hernández, Del Tronco y Sánchez (2009)

12 Los políticos -y no sólo la política-importan. Es cierto que las acciones políticas están mediatizadas por el marco institucional establecido pero finalmente no hay juego democrático si no hay equipos (partidos) y estos últimos a su vez, están compuestos por jugadores (políticos). Un posible abordaje de este problema sería observar la similitud entre las orientaciones ideológicas o valorativas de los mandatarios respecto de sus representados para analizar si dicha cercanía influye indirectamente sobre la confianza de los ciudadanos en sus representantes. De acuerdo con este argumento, una mayor cercanía de las orientaciones políticas de los representantes (las élites parlamentarias, por ejemplo) respecto de los ciudadanos sería una condición para que las políticas públicas representaran un interés similar al del votante mediano, aumentando por consiguiente la confianza en las instituciones representativas (Mateo Díaz, 2006). 
una, y en el papel de los actores partidarios en el proceso político, la otra. Es la calidad del proceso democrático (a través de los cuales dicha política fue incorporada a la agenda, diseñada o implementada) lo que está en juego (Morlino, 2005). Sin embargo, el enfoque más utilizado por la literatura especializada para abordar el tema del desempeño como determinante de las actitudes políticas es aquel que analiza la performance a partir de los efectos de las políticas públicas: índices de crecimiento económico, niveles de inflación, desempleo, déficit fiscal o combate a la pobreza, por mencionar sólo algunos. Tal como sostienen Levine y Molina (2007: 18-19), en este último caso el objeto de estudio ya no es la calidad de la democracia -como procedimiento-, sino el buen gobierno. ${ }^{13}$

A continuación, se presenta la evidencia para cumplir con los dos grandes objetivos del trabajo. En primer lugar, presentar cuáles son los factores que explican la desconfianza de los ciudadanos en las instituciones políticas, para en un segundo momento, analizar si existe alguna relación entre las dimensiones de la calidad de la representación descritas más arriba y la desconfianza en cada una de las instituciones analizadas (partidos, parlamento, gobierno y presidente).

13 Esto no significa que el grado de asociación entre los procedimientos institucionales, el contenido de las políticas y los resultados sea nulo. La calidad institucional es una dimensión previa, cronológicamente anterior, que incide sobre la calidad de la política e indirectamente puede afectar la efectividad del gobierno; sin embargo refiere a una etapa y a un fenómeno diferente. Por su parte, la calidad de la política se constituye en un nexo clave cuyo análisis es ineludible; son los políticos quienes de manera responsable o no, oportunista o no, transforman (o no) los mandatos en leyes; leyes que se convertirán más tarde o más temprano en políticas públicas. 


\section{Evidencia empírica}

La estrategia de análisis se apoya en métodos cuantitativos. Prevé la descripción de los fenómenos analizados a partir de la utilización de técnicas estadísticas (uni y multivariantes, para analizar los factores individuales que afectan la confianza ciudadana. Para la prueba de hipótesis se aplicó un modelo de regresión logit ordinal a través del cual se calcula cómo afectan las distintas variables explicativas la probabilidad de que un ciudadano "modal" o típico aumente o disminuya su desconfianza en las instituciones representativas (Presidente, Gobierno, Parlamento y Partidos Políticos). Los datos utilizados en el modelo provienen de la Onda 2006 del Latinobarómetro -encuesta que analiza el comportamiento político de más de 14 mil ciudadanos de 18 países de América Latina-.

\section{El ciudadano latinoamericano típico y sus actitudes hacia la democracia representativa}

Hablar de un ciudadano latinoamericano típico es un ejercicio de reduccionismo significativo. Sin embargo, a partir de los datos de Latinobarómetro es posible detectar ciertas regularidades respecto de las actitudes de los latinoamericanos hacia la democracia, su funcionamiento efectivo, las instituciones representativas y el Estado de Derecho entre otras importantes dimensiones.

La Tabla 1 muestra las características del ciudadano latinoamericano "más frecuente" respecto de una serie de variables utilizadas como predictores del modelo estadístico presentado más adelante. A partir de estos datos, es posible afirmar que el ciudadano latinoamericano típico tiene actitudes positivas hacia la democracia de tipo liberal-representativo, y hacia un Estado de Derecho, pero en términos generales es muy crítico de la manera en que funciona efectivamente la democracia en el continente. 


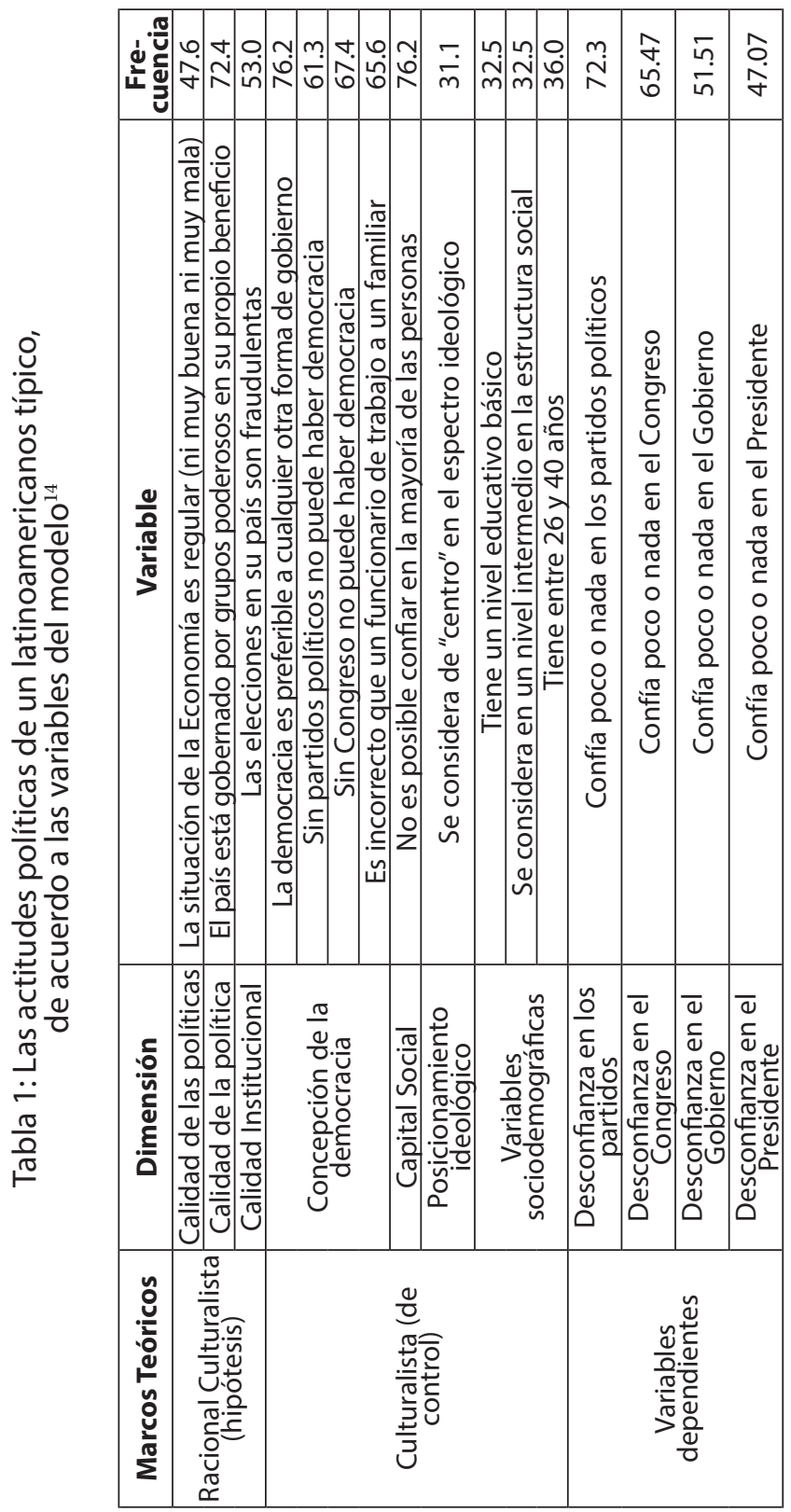

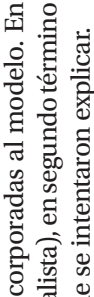

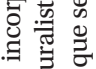

兑吾

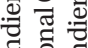

융 융

웜 冚

\&율 융

동.

정

霅

๑

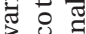

s 켝

잊

त

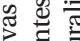

을

证

क्ष की

䔫

氙

음

क

๑ ह

类

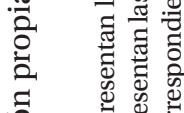

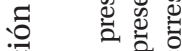

क ष्ठ

풀츄

छ

형 임

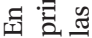


En primer lugar, el ciudadano latinoamericano típico es un adulto joven (tiene entre 26 y 40 años), con un nivel educativo básico, que se autoposiciona en el "medio" de la escala social y se considera ideológicamente "de centro". Este ciudadano tiene una valoración positiva de la democracia como régimen de gobierno: 3 de cada 4 latinoamericanos típicos la consideran el mejor régimen posible más allá de sus problemas. De igual modo, presenta una valoración positiva de los partidos y el congreso, en la medida en que casi dos tercios de ellos consideran que no puede haber democracia si tales instituciones no están presentes. Todo ello supone -en contra de lo planteado por la tesis culturalista- que no existe una predisposición social ni cultural en contra de la democracia ni de su formato representativo, que dispare automáticamente un sentimiento natural de desconfianza institucional. ${ }^{15}$

No obstante estas características, el latinoamericano típico tiende a confiar poco o nada en instituciones como los partidos políticos (72\%) o el parlamento (65\%), y un poco más en el gobierno (49\%) o en el presidente (53\%). Ello significa que hay algo en el funcionamiento de la democracia en América Latina que está creando una brecha entre lo que los ciudadanos esperan (e idealizan, si se quiere) respecto de lo que democracia representativa les está ofreciendo a través de su funcionamiento real.

A partir de los datos de la Tabla 1, es posible detectar algunas posibles explicaciones de esta distancia entre expectativas y realidad. En primer lugar, un $75 \%$ de los latinoamericanos cree que las autoridades no gobiernan a favor de los intereses del pueblo sino de sus propios intereses.

15 La única variable "culturalista" que parece sí tener cierta incidencia es la pauta generalizada de desconfianza de los latinoamericanos para con sus semejantes. Sin embargo, como se verá más adelante con los datos del modelo, esta variable tiene un poder explicativo menor, de acuerdo con lo esperado. 
Ello tiene una incidencia innegable sobre la percepción de la calidad de la política democrática. En segundo lugar, más de la mitad de los latinoamericanos típicos cree que las elecciones en su país son fraudulentas, y que sus resultados no reflejan las preferencias sociales. Vinculado al punto anterior, este hecho reforzaría la posibilidad de que una élite, que gobierna en su propio beneficio, se perpetúe en el poder.

Finalmente, casi la mitad de los ciudadanos latinoamericanos creen que el desempeño de la economía en su país no es del todo bueno ni del todo malo (48\%). Este dato dice poco por sí mismo, por lo que es importante destacar que del $52 \%$ restante, 2 de cada 3 creen que la situación económica es mala o muy mala. Es decir, sólo el 18\% del total considera que la economía funciona bien o muy bien ${ }^{16}$.

Hasta aquí, la evidencia empírica presentada reviste un carácter exploratorio. Es útil para describir tendencias y analizar niveles de asociación entre variables pero no alcanza para establecer con claridad cuáles son los determinantes de la desconfianza institucional, y fundamentalmente, qué diferencias hay entre países, y por institución representativa. Por ello, a continuación se presentan las variables que componen el modelo explicativo y el país en el que cada una de ellas resulta significativa, para poder determinar si las diferencias por país son relevantes como para poner en duda la robustez del modelo para toda la región.

16 Es interesante que pese a ello, y a ser históricamente un continente muy desigual en cuanto a la capacidad de hacer efectivos derechos civiles, políticos y sociales, 6 de cada 10 latinoamericanos creen que es posible nacer pobre y llegar a ser rico a lo largo de la vida. 
Tabla 2: Factores explicativos de la desconfianza institucional en América Latina

\begin{tabular}{|c|c|c|c|c|}
\hline Predictores & $\begin{array}{c}\text { Desconfianza } \\
\text { Partidos }\end{array}$ & $\begin{array}{c}\text { Desconfianza } \\
\text { Congreso }\end{array}$ & $\begin{array}{c}\text { Desconfianza } \\
\text { Gobierno }\end{array}$ & \begin{tabular}{|c} 
Desconfianza \\
Presidente
\end{tabular} \\
\hline Economía regular & $\begin{array}{l}1.515^{* * *} \\
(0.0750)\end{array}$ & $\begin{array}{l}1.402^{* * * *} \\
(0.0712)\end{array}$ & $\begin{array}{l}1.654^{* * * *} \\
(0.0848)\end{array}$ & $\begin{array}{l}1.688^{* * * *} \\
(0.0874)\end{array}$ \\
\hline $\begin{array}{c}\text { Economía mal o } \\
\text { muy mal }\end{array}$ & $\begin{array}{c}\mathbf{2 . 1 8 0 * * *} \\
(0.124)\end{array}$ & $\begin{array}{c}\mathbf{2 . 2 5 0 * * *} \\
(0.130)\end{array}$ & $\begin{array}{c}3.781 * * * \\
(0.209)\end{array}$ & $\begin{array}{c}\mathbf{3 . 8 5 0} * * * \\
(0.212) \\
\end{array}$ \\
\hline $\begin{array}{l}\text { No es posible nacer } \\
\text { pobre y llegar a rico }\end{array}$ & $\begin{array}{l}1.215^{* * *} \\
(0.0471)\end{array}$ & $\begin{array}{l}1.110^{* * * *} \\
(0.0435)\end{array}$ & & $\begin{array}{l}1.110^{* * * *} \\
(0.0388)\end{array}$ \\
\hline $\begin{array}{c}\text { Gob. Autoritario } \\
\text { mejor }\end{array}$ & & & $\begin{array}{l}1.203^{* * *} \\
(0.0563)\end{array}$ & \\
\hline $\begin{array}{c}\text { Indiferente al tipo } \\
\text { de gob. }\end{array}$ & & & $\begin{array}{l}1.155^{* * *} \\
(0.0524)\end{array}$ & \\
\hline $\begin{array}{c}\text { Gob a favor de } \\
\text { poderosos }\end{array}$ & $\begin{array}{l}1.776 * * * \\
(0.0760)\end{array}$ & $\begin{array}{l}1.973 * * * \\
(0.0863)\end{array}$ & $\begin{array}{c}\mathbf{2 . 3 5 5 * * *} \\
(0.101)\end{array}$ & $\begin{array}{c}2.331 * * * \\
(0.0995)\end{array}$ \\
\hline $\begin{array}{c}\text { Democracia mejor } \\
\text { (desacuerdo) }\end{array}$ & $\begin{array}{l}1.309 * * * \\
(0.0688) \\
\end{array}$ & $\begin{array}{l}1.427^{* * *} \\
(0.0750) \\
\end{array}$ & $\begin{array}{l}1.772^{* * * *} \\
(0.0816) \\
\end{array}$ & $\begin{array}{l}1.860^{* * *} \\
(0.0814)\end{array}$ \\
\hline $\begin{array}{c}\text { Democracia } \\
\text { mejor (muy en } \\
\text { desacuerdo) }\end{array}$ & $\begin{array}{c}1.503^{* * *} \\
(0.165)\end{array}$ & $\begin{array}{c}1.677^{* * *} \\
(0.183)\end{array}$ & $\begin{array}{c}2.629 * * * \\
(0.242)\end{array}$ & $\begin{array}{c}2.137^{* * *} \\
(0.182)\end{array}$ \\
\hline $\begin{array}{c}\text { Infringir la ley es } \\
\text { grave }\end{array}$ & $\begin{array}{c}1.027 \\
(0.0482) \\
\end{array}$ & $\begin{array}{c}1.036 \\
(0.0491) \\
\end{array}$ & $\begin{array}{c}0.951 \\
(0.0417) \\
\end{array}$ & $\begin{array}{l}0.922^{*} \\
(0.0401) \\
\end{array}$ \\
\hline $\begin{array}{l}\text { Infringir la ley es } \\
\text { "algo" grave }\end{array}$ & $\begin{array}{l}1.580 * * * \\
(0.0983)\end{array}$ & $\begin{array}{l}1.691^{* * *} \\
(0.104)\end{array}$ & $\begin{array}{l}1.390^{* * * *} \\
(0.0733)\end{array}$ & $\begin{array}{l}1.365^{* * * *} \\
(0.0711)\end{array}$ \\
\hline $\begin{array}{c}\text { Sin partidos, hay } \\
\text { democracia }\end{array}$ & $\begin{array}{l}1.512^{* * * *} \\
(0.0587)\end{array}$ & & & \\
\hline $\begin{array}{c}\text { Sin congreso, hay } \\
\text { democracia }\end{array}$ & & $\begin{array}{l}1.455^{* * *} \\
(0.0590) \\
\end{array}$ & & \\
\hline $\begin{array}{l}\text { Sin privilegios ni } \\
\text { discriminado }\end{array}$ & $\begin{array}{l}0.871^{* *} \\
(0.0544) \\
\end{array}$ & $\begin{array}{l}0.884^{*} \\
(0.0560) \\
\end{array}$ & $\begin{array}{c}1.019 \\
(0.0598) \\
\end{array}$ & $\begin{array}{c}1.091 \\
(0.0625) \\
\end{array}$ \\
\hline $\begin{array}{c}\text { Siente } \\
\text { discriminación }\end{array}$ & $\begin{array}{c}0.969 \\
(0.0454) \\
\end{array}$ & $\begin{array}{c}0.947 \\
(0.0446) \\
\end{array}$ & $\begin{array}{l}0.858^{* * *} \\
(0.0365) \\
\end{array}$ & $\begin{array}{c}0.961 \\
(0.0403) \\
\end{array}$ \\
\hline $\begin{array}{l}\text { No pertenece a } \\
\text { grupo alguno }\end{array}$ & $\begin{array}{c}1.068 \\
(0.0650)\end{array}$ & $\begin{array}{c}1.075 \\
(0.0659) \\
\end{array}$ & $\begin{array}{c}1.033 \\
(0.0553) \\
\end{array}$ & $\begin{array}{l}1.118^{* *} \\
(0.0589) \\
\end{array}$ \\
\hline $\begin{array}{c}\text { Elecciones } \\
\text { fraudulentas }\end{array}$ & $\begin{array}{c}1.486 * * * \\
(0.0580)\end{array}$ & $\begin{array}{c}1.749 * * * \\
(0.0695)\end{array}$ & $\begin{array}{c}\mathbf{2 . 2 7 3 * * * *} \\
(0.0826)\end{array}$ & $\begin{array}{c}2.420 * * * * \\
(0.0869)\end{array}$ \\
\hline $\begin{array}{c}\text { Dar trabajo a un } \\
\text { familiar (incorrecto } \\
\text { y comprensible) }\end{array}$ & $\begin{array}{l}1.111^{* *} \\
(0.0512)\end{array}$ & $\begin{array}{l}1.293^{* * * *} \\
(0.0597)\end{array}$ & $\begin{array}{l}1.274^{* * *} \\
(0.0539)\end{array}$ & $\begin{array}{l}1.290^{* * *} \\
(0.0532)\end{array}$ \\
\hline $\begin{array}{c}\text { Dar trabajo a los se- } \\
\text { guidores (incorrecto } \\
\text { y comprensible) }\end{array}$ & $\begin{array}{l}1.143^{* * * *} \\
(0.0541)\end{array}$ & $\begin{array}{l}1.326^{* * * *} \\
(0.0582)\end{array}$ & $\begin{array}{l}1.276^{* * *} \\
(0.0558)\end{array}$ & $\begin{array}{l}1.342^{* * * *} \\
(0.0533)\end{array}$ \\
\hline
\end{tabular}




\begin{tabular}{|c|c|c|c|c|}
\hline $\begin{array}{c}\text { Dar trabajo a parti- } \\
\text { darios (incorrecto y } \\
\text { comprensible) }\end{array}$ & $\begin{array}{l}1.180^{* * *} \\
(0.0516)\end{array}$ & & $\begin{array}{l}1.114^{* * * *} \\
(0.0457)\end{array}$ & \\
\hline $\begin{array}{c}\text { No se puede confiar } \\
\text { en la mayoría de las } \\
\text { personas }\end{array}$ & $\begin{array}{l}1.349^{* * * *} \\
(0.0587)\end{array}$ & $\begin{array}{l}1.218^{* * *} \\
(0.0536)\end{array}$ & $\begin{array}{l}1.217^{* * *} \\
(0.0511)\end{array}$ & \\
\hline $\begin{array}{l}\text { Ha sido víctima de } \\
\text { delito }\end{array}$ & $\begin{array}{l}1.134^{* * * *} \\
(0.0444) \\
\end{array}$ & $\begin{array}{l}1.103^{* *} \\
(0.0434) \\
\end{array}$ & $\begin{array}{l}1.137^{* * * *} \\
(0.0408)\end{array}$ & $\begin{array}{l}1.133^{* * * *} \\
(0.0401)\end{array}$ \\
\hline $\begin{array}{l}\text { Ed. Primaria } \\
\text { incompleta }\end{array}$ & $\begin{array}{c}1.076 \\
(0.0932)\end{array}$ & $\begin{array}{c}0.968 \\
(0.0855)\end{array}$ & $\begin{array}{c}1.015 \\
(0.0860)\end{array}$ & $\begin{array}{c}0.951 \\
(0.0797)\end{array}$ \\
\hline $\begin{array}{c}\text { Ed. Primaria } \\
\text { completa }\end{array}$ & $\begin{array}{c}0.969 \\
(0.0706) \\
\end{array}$ & $\begin{array}{c}1.009 \\
(0.0743) \\
\end{array}$ & $\begin{array}{c}0.960 \\
(0.0680)\end{array}$ & $\begin{array}{l}0.869^{* *} \\
(0.0610) \\
\end{array}$ \\
\hline $\begin{array}{l}\text { Ed. Secundaria } \\
\text { incompleta }\end{array}$ & $\begin{array}{c}0.999 \\
(0.0748)\end{array}$ & $\begin{array}{c}0.991 \\
(0.0748)\end{array}$ & $\begin{array}{c}1.011 \\
(0.0731)\end{array}$ & $\begin{array}{c}0.898 \\
(0.0644)\end{array}$ \\
\hline $\begin{array}{l}\text { Ed. Secundaria } \\
\text { completa }\end{array}$ & $\begin{array}{c}1.011 \\
(0.0802)\end{array}$ & $\begin{array}{c}1.053 \\
(0.0842)\end{array}$ & $\begin{array}{l}1.334^{* * *} \\
(0.1000)\end{array}$ & $\begin{array}{l}1.247^{* * *} \\
(0.0919)\end{array}$ \\
\hline $\begin{array}{l}\text { Ed. Terciaria } \\
\text { incompleta }\end{array}$ & $\begin{array}{l}0.854^{* *} \\
(0.0632)\end{array}$ & $\begin{array}{l}0.777^{* * * *} \\
(0.0583)\end{array}$ & $\begin{array}{c}1.011 \\
(0.0722) \\
\end{array}$ & $\begin{array}{l}0.888^{*} \\
(0.0625)\end{array}$ \\
\hline $\begin{array}{l}\text { Ed. Terciaria } \\
\text { completa }\end{array}$ & $\begin{array}{l}0.796^{* *} \\
(0.0761)\end{array}$ & $\begin{array}{l}0.822^{* *} \\
(0.0809)\end{array}$ & $\begin{array}{l}1.167^{*} \\
(0.103)\end{array}$ & $\begin{array}{c}0.959 \\
(0.0829)\end{array}$ \\
\hline 26 a 40 años & & $\begin{array}{l}1.159 * * * \\
(0.0561) \\
\end{array}$ & $\begin{array}{l}0.912^{* *} \\
(0.0400) \\
\end{array}$ & $\begin{array}{l}0.863^{* * * *} \\
(0.0374) \\
\end{array}$ \\
\hline 41 a 60 años & & $\begin{array}{l}1.118^{* *} \\
(0.0596)\end{array}$ & $\begin{array}{l}0.797^{* * *} \\
(0.0383)\end{array}$ & $\begin{array}{l}0.781^{* * * *} \\
(0.0372)\end{array}$ \\
\hline 61 y más & & $\begin{array}{l}1.131^{*} \\
(0.0795)\end{array}$ & $\begin{array}{l}0.848^{* * * *} \\
(0.0541)\end{array}$ & $\begin{array}{l}0.822^{* * *} \\
(0.0516)\end{array}$ \\
\hline Mujer & & $\begin{array}{l}0.902^{* * *} \\
(0.0338) \\
\end{array}$ & & $\begin{array}{l}0.933^{* * *} \\
(0.00990) \\
\end{array}$ \\
\hline $\begin{array}{c}\text { Posición en la escala } \\
\text { social }\end{array}$ & $\begin{array}{l}0.966^{* * *} \\
(0.0115)\end{array}$ & $\begin{array}{l}0.944^{* * * *} \\
(0.0114)\end{array}$ & $\begin{array}{l}0.933^{* * *} \\
(0.0101)\end{array}$ & $\begin{array}{c}1.013^{*} \\
(0.00667)\end{array}$ \\
\hline $\begin{array}{c}\text { Posicionamiento } \\
\text { ideológico }\end{array}$ & $\begin{array}{c}0.990 \\
(0.00766) \\
\end{array}$ & $\begin{array}{c}0.997 \\
(0.00781) \\
\end{array}$ & & \\
\hline cut1 & $\begin{array}{c}1.373^{* * *} \\
(0.164)\end{array}$ & $\begin{array}{c}1.962^{* * * *} \\
(0.249)\end{array}$ & $\begin{array}{c}6.011^{* * *} \\
(0.713)\end{array}$ & $\begin{array}{c}5.930^{* * * *} \\
(0.674)\end{array}$ \\
\hline cut2 & $\begin{array}{l}7.896^{* * *} \\
(0.956)\end{array}$ & $\begin{array}{c}11.10^{* * * *} \\
(1.431) \\
\end{array}$ & $\begin{array}{c}38.26^{* * * *} \\
(4.655)\end{array}$ & $\begin{array}{c}29.30^{* * * *} \\
(3.404)\end{array}$ \\
\hline $\begin{array}{c}\text { No. de } \\
\text { Observaciones }\end{array}$ & 10,717 & 10,566 & 13,943 & 14,617 \\
\hline
\end{tabular}

Error estándar en paréntesis ${ }^{* * *} \mathrm{p}<0.01,{ }^{* *} \mathrm{p}<0.05,{ }^{*} \mathrm{p}<0.1$

Fuente: Elaboración propia con datos de Latinobarómetro 2006 (ordered logit analysis) 
Los resultados de los cuatro modelos logit ordenados muestran que el predictor más importante de la desconfianza institucional es el desempeño económico percibido por los ciudadanos. En las cuatro instituciones analizadas (Partidos, Congreso, Gobierno y Presidente) considerar que la situación económica del país es mala o muy mala es el factor de mayor impacto sobre la desconfianza de los ciudadanos (calidad de las políticas). En el caso de los partidos políticos, el congreso y el gobierno, la segunda variable con mayor incidencia sobre la desconfianza es la creencia de los ciudadanos de que el gobierno favorece los intereses de una minoría poderosa y no los de todo el pueblo (calidad de la política), lo que demuestra la importancia que otorgan los latinoamericanos a la forma en la cual es ejercido el poder. En el modelo explicativo de la desconfianza en el presidente, sin embargo, la segunda variable con mayor peso no tiene que ver con el ejercicio del poder sino con su origen: la limpieza de las elecciones (calidad institucional).

En el caso de las variables culturalistas, tener valores prodemocráticos es el factor con mayor incidencia en los niveles de confianza institucional. El hecho de considerar a la democracia como "el mejor régimen de gobierno posible" tanto como creer que su funcionamiento no es posible sin partidos o sin congreso afectan significativamente en la probabilidad de un latinoamericano típico de confiar en las instituciones representativas. Adicionalmente, tender a confiar en los semejantes disminuye la probabilidad de no confiar en las instituciones representativas.

Como hallazgos interesantes (contra-intuitivos aunque esperados de acuerdo con nuestra hipótesis), encontramos que el nivel educativo, el lugar ocupado en la escala social o el auto-posicionamiento ideológico no tienen un impacto significativo sobre la (des)confianza institucional de los ciudadanos latinoamericanos. 
Figura 3: Desempeño económico y desconfianza en los partidos políticos (América Latina 2006)

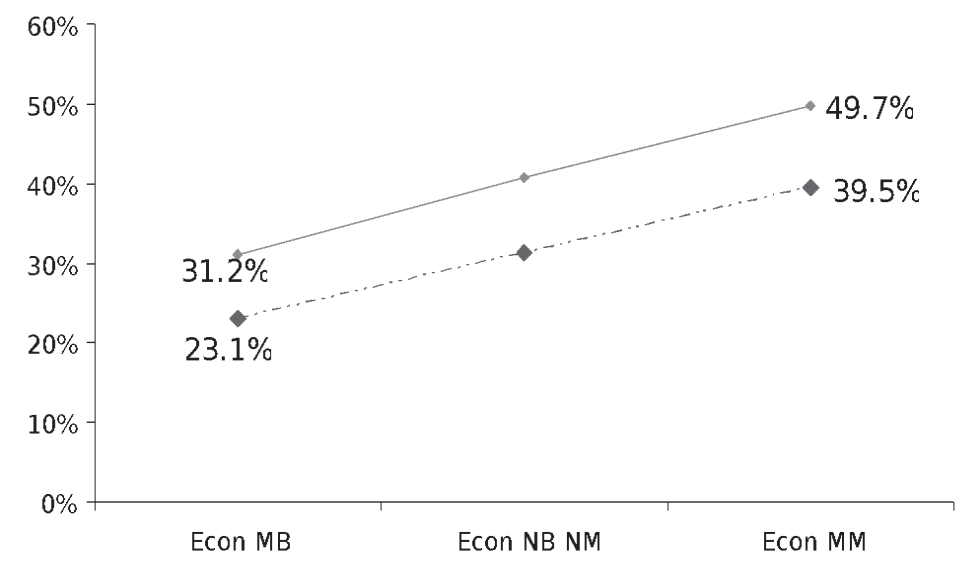

$-\bullet$ Sin partidos no puede haber democracia $\multimap-$ Sin partidos sí puede haber democrac

Fuente: Elaboración propia a partir de los resultados del Ordered Logit Analysis (Datos LB 2006)

La figura 2 muestra cómo cambia la probabilidad de desconfiar en los partidos políticos de un ciudadano latinoamericano típico, a partir de un cambio en su percepción del desempeño económico, controlado por sus valores prodemocráticos. La probabilidad de un ciudadano modal de no confiar en los partidos políticos es del 31.2, puesto que -tal como se vio en la Tabla 1-, este individuo considera que la performance económica es regular y que sin partidos políticos no puede haber democracia. 
Ahora bien, cuando este mismo ciudadano considera que la economía de su país está muy bien, ceteris paribus, ${ }^{17}$ su desconfianza disminuye 9 puntos. Por el contrario, una percepción negativa del funcionamiento de la economía se traduce en un aumento de su desconfianza hasta alcanzar una probabilidad del 39.5, resultados que demuestran la importancia de la calidad de las políticas como factor explicativo de la confianza institucional.

La riqueza del análisis, sin embargo, no se detiene allí. La figura 2 presenta una hallazgo adicional de relevancia. Cuando este mismo ciudadano modal es igual en todo al anterior pero diferente respecto de sus valores democráticos (considera que "sí puede haber democracia sin partidos políticos"), su disposición a desconfiar de los partidos es mayor, independientemente del desempeño de la economía, y crece más drásticamente ante un deterioro de ésta última.

Esto implica, al menos, tres cosas: En primer lugar, que independientemente de los valores del ciudadano (y de su concepción de democracia), una caída en el desempeño económico aumenta la probabilidad de desconfiar en los partidos políticos (17 en promedio). En segundo término, la evidencia muestra que los valores de los ciudadanos tienen incidencia sobre la probabilidad de confiar o no en las instituciones políticas. Cuando un ciudadano tiene valores pro-democráticos tendrá una propensión menor a desconfiar -en este caso de los partidos- que otro cuyos valores son menos cercanos a la democracia representativa (9 en promedio). Finalmente, los datos demuestran que el impacto del desempeño sobre la desconfianza -que es mayor en promedio al impacto de los valores- es mayor en los ciudadanos con valores menos democráticos, quienes tienen

17 Término latino utilizado habitualmente en ciencias sociales que significa: "manteniendo constantes al resto de los factores" 
un nivel de tolerancia menor a los malos desempeños de las instituciones representativas, y eso se manifiesta en su mayor propensión a desconfiar ante una caída en la calidad de las políticas, o para ser más precisos, de sus resultados.

Cuando analizamos desde esta misma perspectiva la confianza de los ciudadanos en el Congreso, el Gobierno y el Presidente, encontramos resultados muy similares (si para el Congreso y el Presidente las gráficas muestran el efecto de la segunda variable más importante).

Figura 4: "Responsiveness" y desconfianza en el congreso (América Latina 2006)

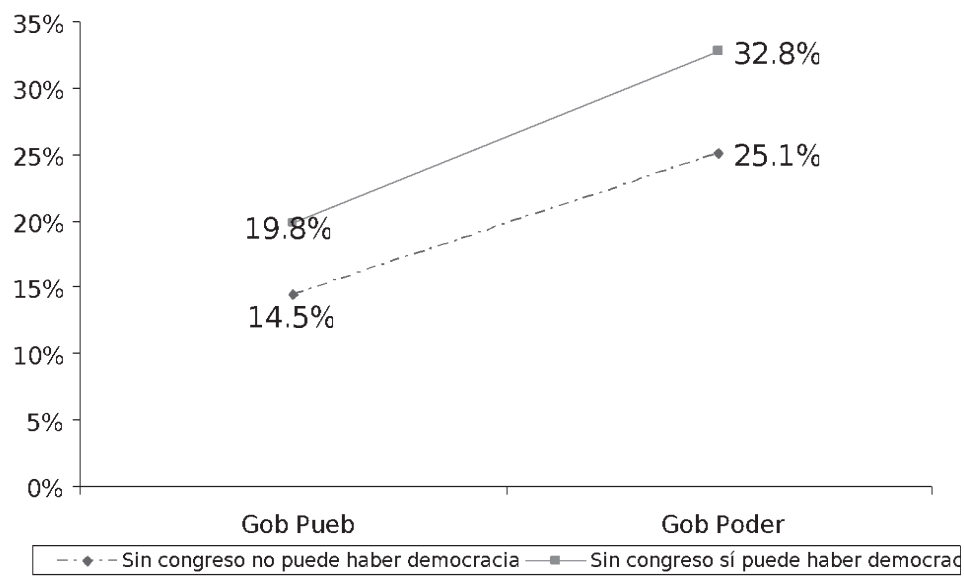

Fuente: Elaboración propia a partir de los resultados del Ordered Logit Analysis (Datos LB 2006)

La figura 4 muestra que la probabilidad de desconfiar en el Congreso tiende a ser mayor para (25.1) para aquellos que consideran que se gobierna a favor de los intereses de una minoría poderosa, respecto de aquellos que creen 
que se gobierna a favor de los intereses del pueblo (14.5). Adicionalmente, este aumento de la desconfianza es aún agudo para aquellos con valores democráticos menos liberales, que consideran posible la democracia, incluso si no hay un parlamento funcionando (32.8). En este caso, se confirma la existencia de una relación inversa entre "calidad del proceso de representación política" -entendida como responsiveness o cumplimiento de los mandatos- $\mathrm{y}$ desconfianza institucional.

Figura 5: Economía y desconfianza en el gobierno (América Latina 2006)

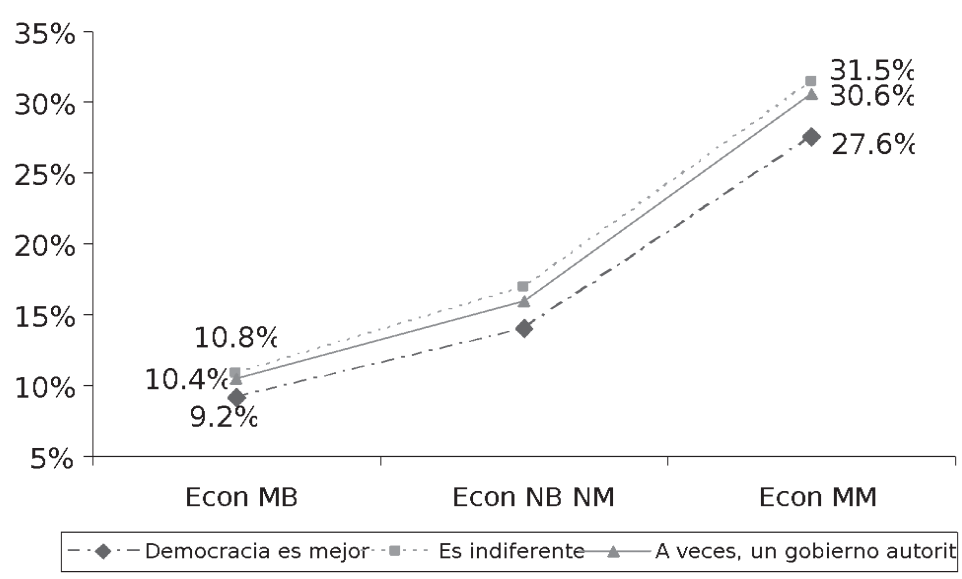

Fuente: Elaboración propia a partir de los resultados del Ordered Logit Analysis (Datos LB 2006)

Como muestran las figuras 4 y 5, en América Latina, los ciudadanos tienden a confiar más en el gobierno y en los presidentes que en los partidos políticos y el parlamento. Ello se ve reflejado en la baja proporción de ciudadanos que manifiesta confiar "nada" en estas instancias. Para el 
caso del gobierno, representa un promedio del 14\%, si bien la probabilidad de que un ciudadano típico confíe "poco" en el gobierno es del $38 \%$.

Sin embargo, estos niveles de desconfianza aumentan a partir de una serie de factores institucionales, políticos y culturales. El más importante de ellos es -al igual que en el caso de los partidos- el desempeño económico. Cuando un latinoamericano típico percibe que la economía funciona mal o muy mal, su nivel de desconfianza aumenta en promedio 18 puntos porcentuales, respecto de aquel que cree que la economía está bien o muy bien.

No obstante, debe destacarse la presencia de variables "culturales" que inciden sobre los niveles de desconfianza en el gobierno. La más relevante es la valoración del régimen democrático. En tal sentido, cuando un ciudadano considera que la democracia "siempre es preferible", sus niveles de desconfianza ante una desmejora del desempeño económico aumentan (18 puntos) pero menos que proporcionalmente que aquellos que consideran que "a veces un régimen autoritario puede ser mejor" (20 puntos) y también para aquellos a quienes "el régimen político vigente les resulta indiferente" (21 puntos).

Finalmente, para el caso del gobierno, los tres hallazgos se comprueban. El impacto de la variable que mide el desempeño institucional (¿las elecciones en su país son libres o fraudulentas?) tiene un impacto sobre la desconfianza mayor a la variable culturalista. En segundo lugar, la valoración de la democracia sí afecta la probabilidad de desconfiar en el presidente. Para un latinoamericano típico que cree que la democracia es mejor más allá de sus problemas, la probabilidad media de "confiar nada" en el presidente es de 15.2, mientras que para quienes están en desacuerdo o muy en desacuerdo, dicha probabilidad en promedio es del 25 y 28 respectivamente. 
Figura 5: Elecciones y desconfianza en el presidente (América Latina 2006)

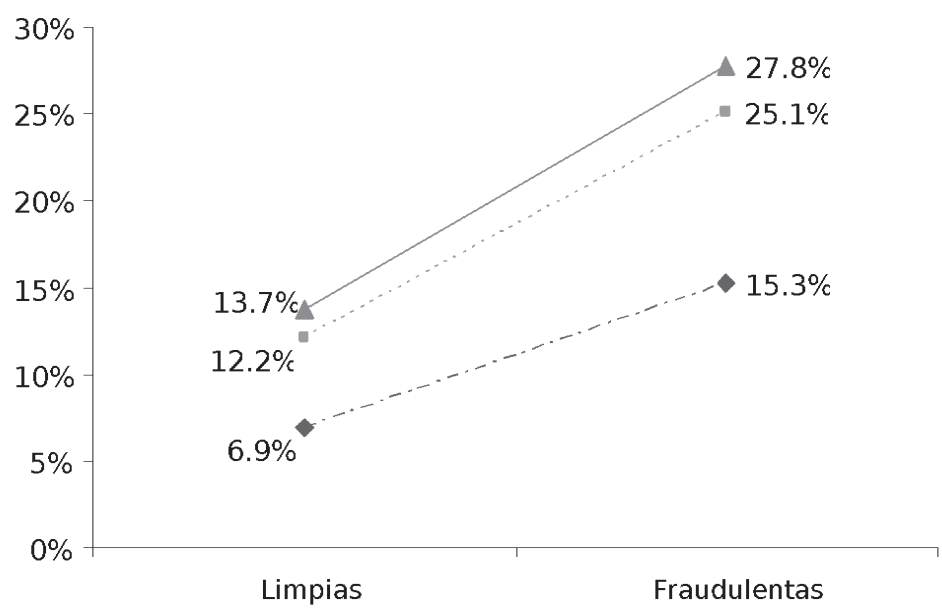

- Democracia es mejor - - En desacuerdo - —- Muy en desacuer

Fuente: Elaboración propia a partir de los resultados del Ordered Logit Analysis (Datos LB 2006)

Por último, una percepción negativa de la calidad institucional (quienes creen que "las elecciones en el país son fraudulentas") tienen un impacto mayor sobre la desconfianza en el presidente para los perfiles menos democráticos; es decir, aquellos que están en desacuerdo (+ 13 puntos) o muy en desacuerdo (+ 14 puntos) con que "la democracia es siempre el mejor régimen de gobierno". Una vez más, los valores prodemocráticos juegan su papel. Aquellos latinoamericanos que consideran a la democracia el mejor régimen de gobierno, están dispuestos a confiar más en el presidente -independientemente de la calidad de las elecciones- que quienes no están de acuerdo con tal 
afirmación. La desconfianza entre los demócratas aumenta en menos de 10 puntos ante un cambio en la calidad de las elecciones, mientras que dicho aumento es de $13 \mathrm{y}$ 14 puntos respectivamente entre los ciudadanos menos democráticos.

\section{Conclusiones}

El análisis de la evidencia empírica muestra con claridad que los factores que más fuertemente explican la desconfianza de los ciudadanos en las instituciones políticas en América Latina son aquellos que miden la calidad del proceso representación. En primer lugar, la variable que mide la percepción de los ciudadanos acerca de la situación económica del país muestra que efectivamente la calidad de los resultados de las políticas es una dimensión central para entender por qué los ciudadanos depositan o no su confianza en determinados actores e instituciones. En este caso, una caída en la percepción positiva del desempeño económico disminuye la confianza de manera significativa en las cuatro instituciones, aunque menos fuertemente en el caso de los partidos políticos, a quienes los ciudadanos parecen no atribuirle la responsabilidad que sí le atribuyen al gobierno en general y al presidente en particular.

En segundo término, la confianza ciudadana depende en buena medida del cumplimiento de los mandatos por parte de los representantes. Aquellos ciudadanos latinoamericanos que consideran que el gobierno representa los intereses de los poderosos, en promedio, tienen una mayor probabilidad de desconfiar de todas las instituciones representativas aquí analizadas, que aquellos que creen que el gobierno representa los intereses del pueblo. Esto demuestra que la ausencia de responsiveness es un predictor fundamental de la desconfianza ciudadana. En tercer lugar, 
debe destacarse la importancia explicativa de la calidad institucional. Quienes perciben que las elecciones en su país son "fraudulentas", expresan una mayor desconfianza (principalmente del gobierno y del presidente, y en menor medida del parlamento y de los partidos) que aquellos que consideran que las elecciones han sido limpias.

Por su parte, hay que destacar también que los valores culturales tienen cierta incidencia sobre la probabilidad de desconfiar de las instituciones representativas pero menor que los indicadores de desempeño. En tal sentido, es la tesis culturalista tradicional que postula la relevancia de los valores autoritarios (el considerar que la democracia "no" siempre es mejor, o creer que la democracia puede funcionar sin parlamento o sin partidos políticos), la que predice con mayor fuerza dicha predisposición. Los enfoques culturalistas contemporáneos, que basan su análisis en la importancia del capital social (expresada en los niveles de confianza interpersonal como predictores de la confianza institucional), o en el cambio en los valores individuales producido por la modernización como causa de la desconfianza no poseen significación estadística, con excepción de algunas categorías, y sólo en algunos países.

En síntesis, el presente trabajo pudo comprobar que: 1) el desempeño -y especialmente el rendimiento económico- tiene mayor impacto sobre la desconfianza que el tipo de valores adquiridos durante el proceso de socialización; 2) las variables de la corriente culturalista -que miden los valores pro-democráticos de los ciudadanos- también inciden en los niveles de confianza, reforzando el efecto del desempeño; y, por lo tanto 3) el impacto del desempeño sobre los niveles de desconfianza en proporcionalmente mayor en los ciudadanos con valores menos democráticos.

Como apuntes para análisis futuros, se expone aquí una paradoja interesante que el presente trabajo - por razones de espacio- no puede desarrollar. A lo largo de las últimas 
décadas, en algunos países, las crisis de representación y confianza ciudadana llevaron al poder a gobiernos "neopopulistas": Fujimori (Perú), Chávez (Venezuela), Evo Morales (Bolivia), Gutiérrez y Correa (Ecuador) ${ }^{18}$ En la mayoría de estos casos, la confianza institucional aumentó a partir de la llegada al poder de estos líderes. A partir de una rendición de cuentas "simbólica" (información y argumentación de sus decisiones) casi permanente (Schedler, 2007), por primera vez en mucho tiempo, los ciudadanos consideran que el gobierno representa los intereses del pueblo y no los de una élite o sectores de poder.

Así, una proporción significativa de estos liderazgos ha mantenido el apoyo y la confianza de los ciudadanos a través del tiempo más allá de las críticas de sus adversarios sobre los efectos nocivos que esta interpelación directa líderpueblo por encima de las instituciones de intermediación puede tener sobre el formato representativo tradicional de la democracia liberal (Weyland, 2004).

¿Representan Chile y Brasil experiencias igualmente exitosas pero institucionalmente diferentes? En ambos casos, la confianza de los ciudadanos ha crecido, pero fundamentalmente en el gobierno y/o en quien ocupa el cargo de presidente (Lula, Lagos), pero no en los partidos ni en el Parlamento (Latinobarómetro 2007, 08 y 09). ¿No será entonces que, como lo muestran los perfiles ciudadanos, están los latinoamericanos más dispuestos a confiar en líderes y/o en instancias capaces de decidir que en instituciones típicamente deliberativas y negociadoras como el congreso o los partidos políticos? Más que nunca, las preguntas están abiertas. Lo que parece estar claro es

18 Una discusión más amplia sobre el concepto de populismo debe darse para este análisis y permitiría quizás incorporar a esta lista a líderes como Néstor Kirchner o Álvaro Uribe. Para este autor, son suficientes los casos presentados más arriba como ilustración del argumento. 
que, más allá de los formatos, es importante representar cabalmente los intereses ciudadanos. La confianza va de la mano del cumplimiento y en esto las instituciones, la política y las políticas son determinantes, pero las últimas llevan algo de ventaja.

\section{Bibliografía consultada}

Almond, G. y S. Verba (1959), The Civic Culture, Princeton, Princeton University Press.

Almond, G., B. Powell Jr., R. Dalton y K. Strom (2002), Comparative Politics Today, Londres, Longman.

Beetham, D. (2007), "Calidad de la democracia: El gobierno de la ley", en C. Cansino e I. Covarrubias, Por una democracia de calidad, México DF, CEPCOM.

Camoes, P.J. y S.M. Méndes (2000), "Winning, Losing and Political Trust Across Generations", versión original presentada en el 72nd Annual Meeting of the Southern Political Science Association, Atlanta GA, 8-11 noviembre.

Catterberg, G. y A. Moreno (2006), “The Individual Bases of Political Trust: Trends in New and Established Democracies", en International Journal of Public Opinion Research 18 (1), pp. 31-48.

Dalton, R. (1999), "Political Support in Advanced Industrial Democracies", en P. Norris, Critical Citizens, New York, Oxford.

Dalton, R. (2005), Citizen Politics, CQ Press.

Easton, D. (1953), The Political System, New York, Alfred A. Knopf, Inc.

-_(1965), A Frameworkfor Political Analysis, Prentice-Hall. (1975), "A Re-Assessment of the Concept of Political Support", en British Journal of Political Science 5 (4), pp. 435-457. 
Eckstein, H. (1988), "A Culturalist Theory of Political Change", en American Political Science Review 82, pp. 789-804. Escaith, H. e I. Paunovic (2004), Reformas Estructurales en América Latina y el Caribe 1970-2000: Índices y notas metodológicas, CEPAL Documento electrónico LC/W.10 Disponible en www.eclac.org.

Fiorina, M. (1981), Retrospective Voting, New Heaven, Yale University Press.

Gambetta, D. (1988), Trust. Making and breaking cooperative relations, Oxford, Basil Blackwell.

Gargarella, R. (2002), Crisis de representación politica, México DF, Fontamara.

Harding, R. (1998), "Trust in Government", en V. Braithwaite y M. Levi (Eds.), Trust and Governance, New York, Russell Sage Foundation.

Holmberg (1999), "Down and down we go: 'Political Trust in Sweden", en P. Norris, Critical Citizens, New York, Oxford.

Inglehart, R. et al. (1996), “Cultural Values, Stable Democracy and Economic Development: A Replay", en American Journal of Political Science 40, pp. 680-696.

Inglehart, R. y Ch. Wezel (2005), Modernization, Cultural Change, and Democracy: The Human Development Sequence, Cambridge, Cambridge University Press.

Klingemann, H.D. (1999), "Mapping political support in the 1990's: A global analysis", en Critical Citizens, New York, Oxford University Press.

Knight, J. (1996), Institutions and Social Conflict, New York, Cambridge University Press.

Listhaug, O. (2006), "Political disaffection and political performance: Norway, 1957-2001", en M. Torcal y J.R. Montero, Political Disaffection in Contemporary Democracies, Londres, Routledge.

Listhaug, O. y M. Wiberg (1995), "Confidence in Political and Private Institutions", en R. Klingemann y D. Fuchs, 
Citizens and the State. Beliefs in Government, Bd. 1, Oxford, Oxford University Press.

Locke, R. (1999), The origins of Trust, en mimeo.

Magalhaes, P. (2006), "Confidence in parliaments: performance, representation and accountability", en M. Torcal y J.R. Montero, Political Disaffection in Contemporary Democracies, Londres, Routledge.

Manin, B. (1998), Los principios del gobierno representativo, Madrid, Alianza Editorial.

Martínez, M.A. (2004), "La representación política y la calidad de la democracia", en Revista Mexicana de Sociología, año 66, No. 4, octubre-diciembre, México DF, pp. 661-710.

McAllister, I. (1999), "The economic performance of Governments", en P. Norris, Critical Citizens, New York, Oxford.

Miller, A. y O. Listhaug (1999), "Institutional trust and political performance", en P. Norris Critical Citizens, New York, Oxford.

Mishler, W. y R. Rose (2001), "What Are the Origins of Political Trust? Testing Institutional and Cultural Theories in Post-Communist Societies", en Comparative Political Studies 34, 1, pp. 30-62.

Mouffe, C. (2007), "La democracia tiene que darles lugar al disenso y la pasión", en http://www.lanacion.com. ar/Archivo/Nota.asp?nota_id=949548.

Newton, K. (1999), "Social and Political Trust in Established Democracies", en P. Norris, Critical Citizens, New York, Oxford.

Newton, K. y P. Norris (2000), "Confidence in public institutions: Faith, Culture, or Performance?", en R. Putnam, S. Pharr y R. Dalton, Dissaffected Democracies, New Jersey, Princeton University Press. 
Nye Jr, J. (1998), “The decline of confident in government", en J. Nye Jr et al., Why people don 't trust in governments Cambridge, Harvard University Press.

O’Donnell, G. (1993), "Estado, democratización y ciudadanía", en Nueva Sociedad 128, Noviembre-Diciembre, pp. 62-87.

- -(1994), "Delegative Democracy", en Journal of Democracy 5, No. 1, enero, National Endowment for Democracy and The Johns Hopkins University Press, pp. 55-69..

O’Donnell, G., J. Vargas Cullel y O.M. Iazzetta (Coords.) (2004), The Quality of Democracy: Theory and Applications, Notre Dame, Notre Dame University Press.

Offe, C. (2005), "Democracy, Disaffection and Institutions: Some Neo-Tocquevillean Speculations", en M. Torcal y J.R. Montero (eds.), Political Disaffection in Contemporary Democracy: Social Capital, Institutions and Politics, Londres, Routledge.

Peters, G. (2003), El Nuevo Institucionalismo en la Ciencia Política, Barcelona, Gedisa.

Payne, M., D. Zovatto y A. Mateos Díaz (2006), La politica importa, Washington, Banco Interamericano de Desarrollo.

Itkin, H. (1985), El concepto de representación, Madrid, Centro de Estudios Constitucionales.

- -(2004), "Representation and democracy: Un uneasy alliance", en Scandinavian Political Studies 27, No. 3.

Powell Jr., B.G., (2004), "Calidad de la democracia: reciprocidad y responsabilidad", en C. César e I. Covarrubias, Por una democracia de calidad, México DF, CEPCOM. Programa De Las Naciones Unidas Para El Desarrollo (2004), La democracia en América Latina: Hacia una democracia de ciudadanas y ciudadanos, Buenos Aires, Aguilar-Altea-Taurus-Alfaguara. 
Przweorski, A. (1995), Democracia y Mercado, Cambridge, Cambridge.

Przeworski, A. y J.M. Maravall (comp.) (2003), Democracy and the rule of law, Cambridge, Cambridge University Press.

Putnam, R.D. (1993), Making democracy work, New Jersey, Princeton University Press.

-_(2000), Bowling Alone, New York, Simon \& Schuster. Putnam, R., S. Pharr y R. Dalton (1999), Dissafected Democracies, New Jersey, Princeton University Press. Rehefeld, A. (2006), “Trustees, Delegates and Gyroscopes: Democratic Justice and the Ethics of Political Representation", trabajo presentado en el Annual Meeting of the American Political Science Association, 30 de agosto-3 de septiembre.

Segatti, P. (2006), "Italy, forty years of political disaffection: a longitudinal exploration", en M. Torcal y J.R. Montero, Political Disaffection in Contemporary Democracies, Londres, Routledge.

Schedler, A. (2007), “¿Qué es la rendición de cuentas?”, en Cuadernos de Transparencia del IFAI, México DF. Shepsle, K. (1986), "Institutional equilibrium and equilibrium institutions", en H. Weisberg (Ed.), Political Science: The Science of Politics, Nueva York, Agathon Press.

Stokes, S., B. Manin y A. Przeworski (1999), Mandates, Accountability and Representation, Cambridge, Cambridge University Press.

Stokes, S. y M. Cleary (2006), Democracy and the culture of skepticism, Russel Sage Foundation.

Temkin, B., R. Salazar y G. Ramírez (2003), Demos: Ciudadanos de la democracia, México DF, Secretaría de Gobernación.

-_(2006), "Political disaffection and democratization history in new democracies", en M. Torcal y J.R. Montero, 
Political Disaffection in Contemporary Democracies, Londres, Routledge.

Torcal, M. y J.R. Montero (2006), "Political disaffection in comparative perspective”, en M. Torcal y J.R. Montero, Political Disaffection in Contemporary Democracies, Londres, Routledge.

Weyland, K. (2004), Releer los populismos, Quito, Centro Andino de Acción Popular.

\section{Datos e información estadística}

Latinobarómetro (1996-97-98-2000-01-02-03-04-06-0607-08 y 09)

World Values Survey (1981-2004): Información disponible en www.worldvaluessurevey.org 